\title{
A Substractive Clustering Based Fuzzy Hybrid Reference Control Design for Transient Response Improvement of PID Controller
}

\author{
Endra Joelianto \& Parlindungan H. Sitanggang \\ Instrumentation and Control Research Group \\ Department of Engineering Physics \\ Bandung Institute of Technology, Bandung 40132, Indonesia \\ E-mail: ejoel@tf.itb.ac.id
}

\begin{abstract}
The well known PID controller has inherent limitations in fulfilling simultaneously the conflicting control design objectives. Parameters of the tuned PID controller should trade off the requirement of tracking set-point performances, disturbance rejection and stability robustness. Combination of hybrid reference control (HRC) with PID controller results in the transient response performances can be independently achieved without deteriorating the disturbance rejection properties and the stability robustness requirement. This paper proposes a fuzzy based HRC where the membership functions of the fuzzy logic system are obtained by using a substractive clustering technique. The proposed method guarantees the transient response performances satisfaction while preserving the stability robustness of the closed loop system controlled by the PID controller with effective and systematic procedures in designing the fuzzy hybrid reference control system.
\end{abstract}

Keywords: fuzzy systems; hybrid reference control; PID controller; substractive clustering; transient response.

\section{$1 \quad$ Introduction}

Hybrid Reference Control (HRC) has been developed by Joelianto and Williamson [1][2], and Joelianto [3] to improve transient response of stabilized closed loop control systems and to reduce limitations intrinsic in one- degreeof-freedom controller by means of reference signal (set-point) manipulation based on linear impulsive differential equations. The HRC has been successfully improving the transient response performances (rise time, maximum overshoot, and settling time) or deadbeat response at a specified time of the conventional controlled systems. By using HRC, the transient response performances can be designed independently without affecting the stability properties of the closed loop systems. The HRC system is a decision system added to the conventional closed loop systems to manipulate the reference signal (set-point) of feedback control systems based on occurrences of events. The block diagram of the HRC system is shown in Figure 1. In HRC method,

Received February $24^{\text {th }}, 2009$, Revised August $17^{\text {th }}, 2009$, Accepted for publication October $20^{\text {th }}, 2009$. 
the main goal is the transient response performance improvement of the closed loop control systems for given default reference signal values. During the transient response to reach the default reference signal, the control signal is manipulated as a result of applying temporary reference signals in order to speed up or to slow down the speed of the transient response performances. This can be achieved by increasing or decreasing the control signal by means of changing the reference signal appropriately and then returning the reference signal to the default reference signal value when the output is sufficiently close to the default value.

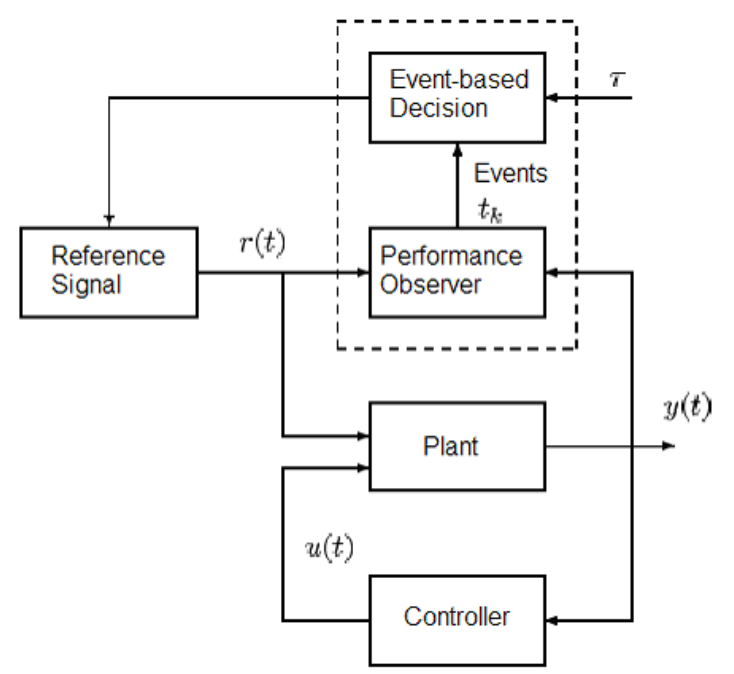

Figure 1 Diagram block of hybrid reference control (HRC).

As in Joelianto and Williamson [1], the events $\left(t_{k}, k=1,2, \cdots, m-1\right)$ are generated by partitioning the error between the default reference signal and the output of the controlled system by a performance observer. The initiation to change the reference signal is initiated by an enabled event when the error is considered to be sufficiently large. The event-based decision system then sends a sequence of temporary reference signals to the closed loop system at a fixed time interval $\left(\tau=t_{k}-t_{k-1}\right)$. When the output of the controlled system enters the specified tolerance of error band of the default reference value, a disable event $\left(t_{m}\right)$ is generated that returns the reference signal to its default reference value. The process is repeated on the occurrences of enable events. In conventional feedback designs, the goal is to seek an appropriate gain/control feedback that stabilizes the closed loop system and achieves some required transient performances. On the other hand, the objective of the HRC design is to find the 
appropriate temporary reference signal that will improve the transient response of the stabilized closed loop control. As a result, the combination of HRC and conventional feedback systems leads to a better control strategy with twodegree-of-freedom controller configuration.

Fuzzy logic controllers are knowledge based or rule based controllers which are inferred from human knowledge or experience on systems. In fuzzy control systems Zadeh [4] and Wang [5], the goal is to put human knowledge into a controller in a systematic, efficient and analyzable order. In the case of PID controllers, it is known that operators play important role in order to achieve good PID controllers by means of applying proper tuning to dealt with conflicting behaviour requirements of the closed loop or by manipulating the input of the controlled process manually. Many published papers mainly focussed on the selection of the three parameters of the PID controller as the application of fuzzy system in mimicking the knowledge of the operators, to illustrate Misir and Malki [6], and Mohan and Sinha [7]. However, there has been little attention to implement fuzzy logic to perform a similar way to an expert operator who suppresses overshoot by either increasing or decreasing the input of the controlled process. Yokogawa [8][9] has proposed a fuzzy logic system based on the information of the process dynamics to reduce overshoot. This method has been one successful example of the application of fuzzy logic in control system design as reported by Chiu [10].

Joelianto and Tansri [11] has proposed a fuzzy logic based HRC system that mimics the way of operator in deciding the magnitude of the input to the controlled process in achieving the specified transient response performance of the interest process variables. Specifically, the adjustment of the input of the controlled process by the output of the PID controller can be produced by the manipulation of the reference signal by means of a fuzzy logic system designed by using the well known fuzzy method Takagi and Sugeno [12]. The stability of the closed control system is maintained by the PID controller with the three PID parameters are selected by using the well known tuning methods Ziegler and Nichols [13]. Other tuning methods discussed in Smith and Corripio [14], Coughanowr [15] and Åström and Hägglund [16] can also be applied to the PID controller. The diagram block of the fuzzy HRC system with the PID controller developed in Joelianto and Tansri [11] is shown in Figure 2. The fuzzy logic system processes the error signal $(e(t))$ which is the difference between the output of the controlled system and the default reference signal and its derivative $(\dot{e}(t))$ in order to decide an appropriate signal that changes the default reference signal during the transient response. 


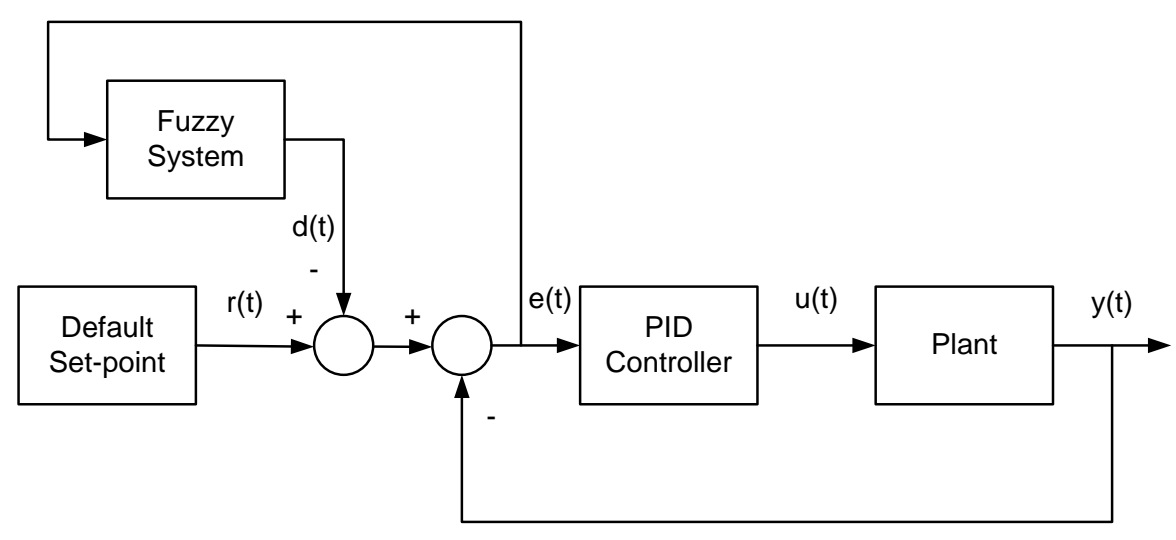

Figure 2 Block diagram of substractive clustering based fuzzy HRC and PID controller.

The fuzzy based HRC starts sending signals $(d(t))$ that manipulate the default reference signal $(r(t))$ when an enable event $t_{k}$ is detected by the performance observer embedded in the fuzzy system. This event informs the performance observer that the deviation of the closed loop system output $(y(t))$ to the default reference signal $(r(t))$ is bigger than the prescribed tolerance $\delta$ such that

$$
|e(t)|=|y(t)-r(t)| \geq \delta .
$$

The fuzzy system sends the reference signal $(d(t))$ either continuously or in a predefined time interval $(\tau)$ until the performance observer detects another disable event where the error of the closed loop system is now entering the allowable tolerance defined by

$$
|e(t)|=|y(t)-r(t)|<\delta
$$

When this event is detected, the fuzzy system then stops sending reference signals $(d(t))$ and the reference signal returns to the default reference signal $(r(t))$ by sending the signal $d(t)=0$. In developing the fuzzy HRC system proposed in [11], the main problem is how to obtain the appropriate membership functions of the fuzzy system effectively in shorter time process design as it was time consuming in carrying out the trial error method. This technique is also inadequate for the requirement of an implementation using adaptive strategies to deal with plant parameters changes. In this paper, the substractive clustering technique Chiu [17] is applied to find the membership 
functions of the fuzzy HRC system with less number of rules and minimum amount of computational time. The substractive clustering method is applied to data consist of error signal $(e(t))$, derivative error signal $(\dot{e}(t))$ and the reference signal $(d(t))$. The proposed method is then referred to as a substractive clustering based fuzzy HRC system.

\section{Substractive Clustering Estimation}

Subtractive clustering developed by Chiu [17] is known as a fast, one-pass algorithm to estimate the number and the centers of clusters in a given data set. It is useful when there is no clear idea how many clusters needed for a given set of data. The resulted cluster estimates can then be used to initialize iterative optimization-based clustering methods and model identification methods. Computational problem in subtractive clustering is solved by using data points as the candidates for cluster centers. This leads to the computation is proportional to the problem size rather than the problem dimension. Although, the actual cluster centers are not necessarily located at one of the data points, in most cases, it gives a good approximation.

Let us consider a collection of $n$ data points $\left\{x_{1}, x_{2}, \cdots, x_{n}\right\}$ in a $M$ dimensional space. Without loss of generality, it is also assumed that the data points have been normalized in each dimension so that they are bounded by a unit hypercube. In substractive clustering Chiu [17], each data point may be considered as a possible cluster center. A measure of the potential of data point $x_{i}$ is defined as follows

$$
P_{i}=\sum_{j=1}^{n} e^{-\left(\frac{2}{r_{a}}\right)^{2}\left\|x_{i}-x_{j}\right\|^{2}}
$$

where $\|\bullet\|$ denotes Euclidian distance and $r_{a}>0$ is a constant. Equation (3) shows that the measure of the potential for a data point is a function of its distances to all other data points. A data point with many neighboring data points will have a high potential value. The constant $r_{a}$ denotes the radius defining a neighborhood; where data points outside this radius have little influence on the potential. The potential of every data point has to be computed using (3). The first cluster center is then chosen from the data point which has the highest potential. 
Let $x_{1}^{*}$ and $P_{1}^{*}$ be the location of the first cluster center and its corresponding potential value respectively. The potential of each data point $x_{1}^{*}$ is then revised by using the potential equation formula

$$
P_{i} \Leftarrow P_{i}-P_{1}^{*} e^{-\left(\frac{2}{r_{b}}\right)^{2}\left\|x_{i}-x_{1}^{*}\right\|^{2}}
$$

where $r_{b}>0$ is a constant. Equation (4) shows an amount of potential from each data point as a function of its distance from the first cluster center. The data points near the first cluster center will have greatly reduced potential, and it will not be selected as the next cluster center. The constant $r_{b}$ denotes the radius defining the neighborhood which will have measurable reductions in potential. To avoid obtaining closely spaced cluster centers, $r_{b}$ is set to be greater than $r_{a}$, a good choice is $r_{b}=1.5 r_{a}$ Chiu [17] or $r_{b}=1.25 r_{a}$ Chiu [18].

Next, the potentials of all data points are revised by using equation (4), the data point with the highest remaining potential is chosen as the second cluster center. The process is then continued until the $k^{\text {th }}$ cluster center has been chosen, the potential of each data point is redefined by the potential equation

$$
P_{i} \Leftarrow P_{i}-P_{k}^{*} e^{-\left(\frac{2}{r_{b}}\right)^{2}\left\|x_{i}-x_{k}^{*}\right\|^{2}}
$$

where $x_{k}^{*}$ denotes the location of the $k^{\text {th }}$ cluster center and $P_{k}^{*}$ is its corresponding potential value. The process of acquiring new cluster center and revising potentials is repeated until the remaining potential of all data points falls below some fraction of the potential of the first cluster center $P_{1}^{*}$. In addition to this criterion for ending the clustering process are criteria for accepting and rejecting cluster centers that help to avoid marginal cluster centers. The algorithm of substractive clustering in finding new cluster centers, revising potentials and repeating the process until the potential value is below the acceptance value considered as significant effects to the final clustering results can be found in Chiu [17][18]. For a problem of $c$ clusters and $n$ data points, Hammouda and Karray [19] has shown the required number of calculations is:

$$
N_{\text {calculation }}=\underset{\text { first.cluster }}{n^{2}}+\underset{\text { remainder.cluster }}{(c-1) n}
$$


Equation (6) shows that the number of calculations is independent to the dimension of the problem. In general, the number of computations is in the range of few ten thousands.

Although the number of clusters (or rules) is automatically determined by the method, it should be noted that the user specified parameter $r_{a}$ (the radius of influence of a cluster center) strongly affects the number of clusters that will be generated. A large $r_{a}$ generally results in fewer clusters with a coarser model, while a small $r_{a}$ can produce excessive number of clusters and a model that does not generalize well (by over-fitting the training data). Therefore, the constant $r_{a}$ acts as a tuning parameter of the desired resolution of the model, which can be chosen based on the resultant complexity and generalization ability of the model. It is clear that choosing $r_{a}$ very small or very large will result in a poor accuracy because if $r_{a}$ is chosen very small the density function will ignore the effect of neighboring data points; while if taken very large, the density function will take into account all the data points in the data space. Chiu [18] suggests the good value of $r_{a}$ is between 0.2 and 0.5 , while Hammouda and Karray [19] shows that a value of $r_{a}$ between 0.4 and 0.7 is adequate.

To extract fuzzy rules from data, the data are separated into groups according to their respective classes. In [17][18], subtractive clustering is then applied to the combined input/output space of each group of data individually for identifying each class of data, i.e. each data point $x_{i}$ is a vector that contains both input and output values. Each obtained cluster center is in essence a prototypical data point that represents a characteristic input/output behavior of the system. Consider a set of $m$ cluster centers $\left\{x_{1}^{*}, x_{2}^{*}, \cdots, x_{m}^{*}\right\}$ found in a $M$ dimensional space and divide the space into $N$ dimensions correspond to input variables and the last $M-N$ dimensions correspond to output variables. Each vector $x_{i}^{*}$ is decomposed into two component vectors $y_{i}^{*}$ and $z_{i}^{*}$, where $y_{i}^{*}$ contains the first $N$ elements of $x_{i}^{*}$ from the coordinates of the cluster center in input space and $z_{i}^{*}$ contains the last $M-N$ elements from the coordinates of the cluster center in output space. To identify the behavior of the system, each cluster center $x_{i}^{*}$ may be translated into a fuzzy rule that can be represented by the rule: 
Rule $i$ :

If $\left\{\right.$ input is near $\left.y_{i}^{*}\right\}$ then output is near $\left.z_{i}^{*}\right\}$.

Given an input vector $y$, the degree of fulfillment of $y$ near $y_{i}^{*}$ is assigned as follows

$$
\mu_{j}=e^{-\left(\frac{2}{r_{a}}\right)^{2}\left\|y-y_{j}\right\|^{2}}
$$

The output vector $z$ is calculated by using the formula

$$
z=\left[\sum_{i=1}^{m} \mu_{i} z_{i}^{*}\right] \div\left[\sum_{i=1}^{m} \mu_{i}\right]
$$

Equation (8) shows the computational model in terms of a fuzzy inference system employing traditional fuzzy if-then rules. Each rule can be written in the more familiar form:

If $Y_{1}$ is $A_{i 1} \& Y_{2}$ is $A_{i 2} \& \ldots$ then $Z_{1}$ is $B_{i 1} \& Z_{2}$ is $B_{i 2} \ldots$

where $Y_{j}$ is the $j^{\text {th }}$ input variable and $Z_{j}$ is the $j^{\text {th }}$ output variable; $A_{i j}$ is an exponential membership function in the $i^{\text {th }}$ rule associated with the $j^{\text {th }}$ input and $B_{i j}$ is a membership function in the $i^{\text {th }}$ rule associated with the $j^{\text {th }}$ output.

For the $i^{\text {th }}$ rule, which is represented by cluster center $x_{i}^{*}, A_{i j}$ is selected as an exponential membership function given by

$$
A_{i j}\left(Y_{j}\right)=\exp \left\{-\frac{1}{2}\left(\frac{Y_{j}-y_{i j}^{*}}{\sigma_{i j}}\right)^{2}\right\}
$$

and $B_{i j}$ is any symmetric membership function with the center around $z_{i j}^{*}$, where $y_{i j}^{*}$ and $z_{i j}^{*}$ are the $j^{\text {th }}$ element of $y_{i}^{*}$ and $z_{i}^{*}$ respectively. The value of $\sigma_{i j}$ is set to $\sigma_{i j}=r_{a}^{2} / 8$ [18]. The method of calculation is similar to an inference system that implements the AND operator with multiplication and it uses the degree of fulfilment of the rule to weight the consequent of each rule. The value of the final output is obtained by weighting average of all the consequents. The rules can be optimized by using available optimization 
methods. One simple approach applied by Chiu [18] is by seting the consequent parameter $z_{i j}^{*}$ as a linear function of the input variables as follows

$$
z_{i j}^{*}=G_{i j} y+h_{i j}
$$

where $G_{i j}$ is an $N$-element vector of coefficients and $h_{i j}$ is a scalar constant. In this case, the if-then rules turn out to be the Takagi-Sugeno type. The optimization of $G_{i j}$ and $h_{i j}$ in all consequent equations, given a set of rules with fixed premise membership functions, is a linear least-squares estimation problem Takagi and Sugeno [12]. This approach has been used by Chiu [17] to optimize the obtained rules from the subtractive clustering method. The optimization is only carried out in the coefficients of the consequent equations which lead to less computational complexity.

In this paper, the substractive clustering technique developed by Chiu [17] which is available in the Fuzzy Logic Toolbox of MATLAB [20] is used to find the number and the value of the membership functions of fuzzy based HRC. The cluster estimation is obtained by using the subclust function. The genfis 2 function then generates a Sugeno-type fuzzy inference system that models the data behavior. There are three parameters to be entered to the functions:

1. Quash factor $\left(s_{f}\right)$ is used to multiply the radii values that determine the neighborhood of a cluster center. It is to quash the potential for outlying points to be considered as part of that cluster, $r_{b}=s_{f} r_{a}$

2. Accept ratio $\left(\varepsilon_{1}\right)$ : A data point $x_{k}^{*}$ will be accepted as a cluster center if the corresponding potential $P_{k}^{*}$ satisfies $P_{k}^{*}>\varepsilon_{1} P_{1}^{*}$

3. Reject ratio $\left(\varepsilon_{2}\right)$ : A data point $x_{k}^{*}$ will be rejected as a cluster center if the corresponding potential $P_{k}^{*}$ satisfies $P_{k}^{*}<\varepsilon_{2} P_{1}^{*}$

\section{Stability of Closed Loop System}

To show stability of the closed loop system, it is necessary to recall the structure of the proposed control design as shown in Figure 1. The proposed method consists of three main parts: the event generation, the fuzzy decision rule and the PID controller. The PID controller works on the continuous ouput signal but the fuzzy decision is activated when there occur the event generated by the condition in equation (1) and (2). In this case, the equation (1) and (2) have been selected such that the equation (1) generates an enabled event then 
followed by timed events based on the sampling time of the system as shown in Figure 3. The strategy has been selected such that the modified reference signal will have pattern that will guarantee the reference signal always returns to the default reference signal. The reference signal sequence is defined as follows.

$$
\begin{aligned}
& r(t)=\left\{\begin{array}{c}
r_{d}\left(t_{k}\right) \\
r_{0}\left(t_{k}+0 \tau\right)=r_{d}\left(t_{k}\right)-d_{k}(0 \tau) \\
r_{1}\left(t_{k}+1 \tau\right)=r_{0}\left(t_{k}+1 \tau\right)-d_{k}(1 \tau) \\
\vdots \\
r_{p-2}\left(t_{k}+(p-2) \tau\right)=r_{p-3}\left(t_{k}+(p-2) \tau\right)-d_{k}((p-2) \tau) \\
r_{p-1}\left(t_{k}+(p-1) \tau\right)=r_{p-2}\left(t_{k}+(p-1) \tau\right)-d_{k}((p-1) \tau)=r_{d}(t)
\end{array} ;\right. \\
& i=0,1, \cdots, p-1
\end{aligned}
$$

where $k, \tau$ and $d_{k}(i \tau)$ denote the enabled event at time $t_{k}$ when the ouput satisfies (1), the sampling time and the reference signal are initiated by the enable event at time $t_{k}$ respectively and $p$ is a finite number denotes the number of reference signal changes. The value of $p$ is unknown and is influenced by the real part of the eigenvalues of the closed loop system matrix and the sampling time $\tau$.

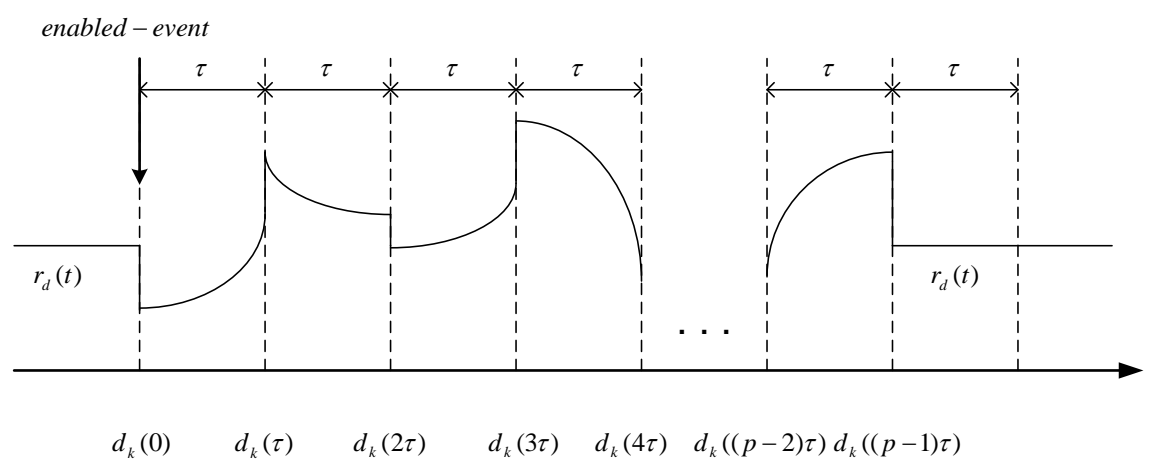

Figure 3 An enabled event followed by timed events [21].

The admissible reference signal for the fuzzy HRC can be defined as follows.

\section{$\underline{\text { Definition }}$}

The reference signals $d_{k}(i \tau)$ are said to be admissible reference signals if 


$$
d_{k}(i \tau) \in \Lambda \text { for } i=0,1, \cdots, p-1
$$

where $\Lambda$ is a set of reference signals which fulfills the condition (11).

In order to analyze the closed loop system, the PID controller is expressed as a full state feedback control law. Consider a single input single output linear time invariant plant described by the state space equation

$$
\begin{aligned}
& \dot{x}(t)=A x(t)+B u(t) \\
& y(t)=C x(t)
\end{aligned}
$$

where the states $x \in R^{n}$ are the solution of (12), the control signal $u \in R^{1}$ is assumed to be the output of a PID controller with input $y \in R^{1}$. The PID controller for regulator problem is of the form

$$
u(t)=K_{1} \int_{0}^{t} y(t) d(t)+K_{2} y(t)+K_{3} \frac{d}{d t} y(t)
$$

which is an output feedback control system and $K_{1}=K_{p} / T_{i}, K_{2}=K_{p}$, $K_{3}=K_{p} T_{d}$ of which $K_{p}, T_{i}$ and $T_{d}$ denote proportional gain, time integral and time derivative of the well known PID controller respectively. The control law (13) is expressed as a state feedback law using (12) by differentiating the plant output $y$ as follows

$$
\begin{aligned}
& y=C x \\
& \dot{y}=C A x+C B u \\
& \ddot{y}=C A^{2} x+C A B u+C B \dot{u} .
\end{aligned}
$$

This implies that the derivative of the control signal (13) may be written as

$$
\left(1-K_{3} C B\right) \dot{u}-\left(K_{3} C A^{2}+K_{2} C A+K_{1} C\right) x-\left(K_{3} C A B+K_{2} C B\right) u=0
$$

Using the notation $\hat{K}$ as a normalization of $K$, this can be written in more compact form

$$
\hat{K}^{T}=\left[\begin{array}{lll}
\hat{K}_{1} & \hat{K}_{2} & \hat{K}_{3}
\end{array}\right]^{T}=\left(1-K_{3} C B\right)^{-1}\left[\begin{array}{lll}
K_{1} & K_{2} & K_{3}
\end{array}\right]^{T}
$$


or $\hat{K}^{T}=c K^{T}$ where $c$ is a scalar. This control law is then given by

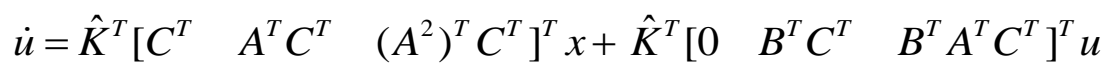

Denote $\quad K_{x}=\hat{K}^{T}\left[\begin{array}{lll}C^{T} & A^{T} C^{T} & \left(A^{2}\right)^{T} C^{T}\end{array}\right]^{T} \quad$ and $K_{u}=\hat{K}^{T}\left[\begin{array}{lll}0 & B^{T} C^{T} \quad B^{T} A^{T} C^{T}\end{array}\right]^{T}$, the block diagram of the control law (16) is shown in Figure 4.

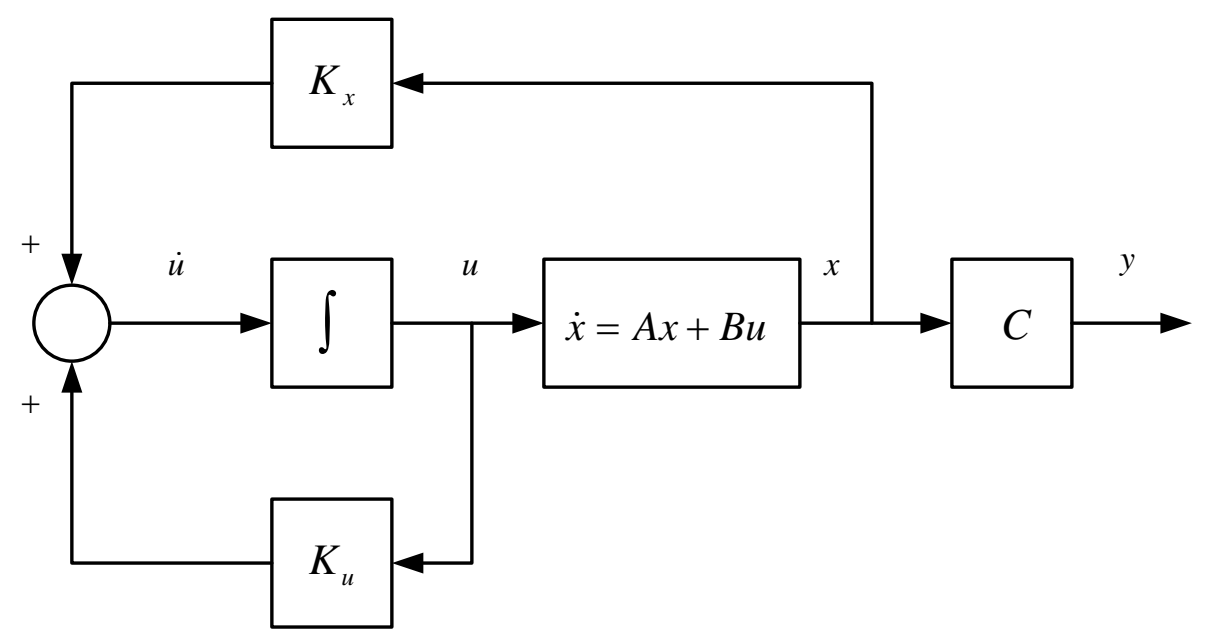

Figure 4 Block diagram of state space representation of PID controller.

Equation (16) represents an output feedback law with constrained state feedback. That is, the control signal (13) may be written as

$$
u_{a}=K_{a} x_{a}
$$

where

$$
\begin{aligned}
& u_{a}=\dot{u}, x_{a}=\left[\begin{array}{l}
x \\
u
\end{array}\right] \\
& K_{a}=\left[\begin{array}{ccc}
C^{T} & A^{T} C^{T} & \left(A^{2}\right)^{T} C^{T} \\
0 & B^{T} C^{T} & B^{T} A^{T} C^{T}
\end{array}\right] \hat{K}
\end{aligned}
$$

The augmented system equation is obtained from (12) and (18) as follows

$$
\dot{x}_{a}=A_{a} x_{a}+B_{a} u_{a}
$$


where

$$
A_{a}=\left[\begin{array}{cc}
A & B \\
0 & 0
\end{array}\right] ; B_{a}=\left[\begin{array}{l}
0 \\
1
\end{array}\right]
$$

Equation (17), (18) and (19) show that the PID controller can be viewed as a state variable feedback law for the original system augmented with an integrator at its input. Once the parameters of the PID controller are selected, the full state feedback gain $K_{a}$ can be calculated using equation (18) and the closed loop system is given by

$$
\dot{x}_{a}=\left(A_{a}+B_{a} K_{a}\right) x_{a}=A_{c l} x_{a} ; A_{c l} \equiv A_{a}+B_{a} K_{a}
$$

The closed loop system is asymptotically stable if the eigenvalues of $A_{c l}$ are strictly Hurwitz.

The closed loop system stability condition can now be summarized as follows.

\section{Theorem}

Consider the fuzzy hybrid reference control in Figure 2. Suppose the parameters of the PID controller are selected such that the closed system (20) is asymptotically stable. If the reference signals generated by the fuzzy decision are admissible reference signals then the closed loop system is asymptotically stable and

$$
\lim _{t \rightarrow \infty} e(t)=0 \text { as } r(t)=r_{p-1}(t+(p-1) \tau)=r_{d}(t) ; i=0,1, \cdots, p-1
$$

where $e(t) \equiv r(t)-y(t)$ for all $t \geq t_{k}+(p-1) \tau$.

\section{Proof}

Recall the asymptotic stability in the equation (20) guarantees that the state error of the closed loop system goes asymptotically to zero as $t \rightarrow \infty$ irrespective to the applied reference signal. The convergence of the output to the default reference signal is fulfilled by the pattern of the reference signal (11). The complete proof involving the concept of internal model principle (IMP) [22] and the principles of the hybrid reference control method can be found in [21].

$\nabla \nabla \nabla$

It has been shown in [21] that proper selection of the implemented reference signal will improve the settling time to the default reference signal. However, 
there is no assurance that the magnitude of the maximum overshoot and the control signal will be lower compared to the original closed loop system. In this case, the generation of the data used in the substractive clustering algorithm that produce faster settling time and lower magnitude of maximum overshoot and control signal is very important in order to guarantee the same result in the PID - fuzzy HRC.

\section{Transient Response Improvement of PID Controller}

Based on the conventional HRC in Joelianto and Williamson [1][2][21] and Joelianto [3] and by using the standard PID, data are generated by requiring that the output responses of the closed loop system are within specified tolerances of the desired transient response performances by changing the reference signals during transient response. The plant which is a first order system with delay time is given by the following transfer function

$$
G(s)=\frac{5 e^{-30 s}}{s^{2}+9 s+10}
$$

The stability of the closed loop system is controlled by the standard PID controller of the form

$$
u(t)=K_{p} e(t)+\frac{K_{p}}{T_{i}} \int_{0}^{t} e(t) d t+K_{p} T_{d} \frac{d e(t)}{d t}
$$

where the parameters are selected by using the well known Ziegler-Nichols tuning method developed by Ziegler and Nichols [13]. The obtained PID parameters are as follow: $K_{p}=0.05, T_{i}=0.6$ second, $T_{d}=15$ second.

In this paper, the transient response specification is maximum overshoot $\left(\bar{M}_{p}\right)$. Data are a collection of transient responses that satisfy the specification as shown in Figure 3. The data consist of error $(e(t))$, derivative error $(\dot{e}(t))$ and the reference signal $(d(t))$ that manipulates the default reference signal $(r(t))$. The data are processed as follows:

- Reject error signal which has value below steady state error $\left(\varepsilon_{a}\right)$

- Accept error signal which has value above $\varepsilon_{a}$ for substractive clustering data

- Reduce zero reference signal data in order to avoid high concentration 
- Equate the number of row between error signal, derivative error signal and reference signal

Next, arrange the data into matrix $X$ as follows:

$$
X=\left[\begin{array}{lll}
X_{1} & X_{2} & X_{3}
\end{array}\right]
$$

where $X_{1}$ : error signal column matrix, $X_{2}$ : derivative error signal column matrix, $X_{3}$ : reference signal column matrix.

The algorithm of substractive clustering based fuzzy-HRC is summarized as follow:

1. Select parameters of PID controller $\left(K_{p}, T_{i}, T_{d}\right)$ and the event tolerance $\delta$

2. Generate data manually by removing the fuzzy system: error signal, derivative error signal and reference signal using Figure 2.

3. Set allowable maximum overshoot $\left(\bar{M}_{p}\right)$ and allowable steady state error $\left(\varepsilon_{a}\right)$

4. Reject data greater than $\bar{M}_{p}$ and less than $\varepsilon_{a}$

5. Select data range of $\left[\begin{array}{lll}X_{1} & X_{2} & X_{3}\end{array}\right]$ and parameters of subclust function $\left(s_{f}, \varepsilon_{1}, \varepsilon_{2}\right)$

6. Arrange data into input data (error signal and derivative error signal) and output data (reference signal)

7. Use the input data, output data and parameters of subclust function into genfis2 function

In the example, the parameters are selected as follow:

Transient response: $\bar{M}_{p}=15 \%$ and $\varepsilon_{a}=2 \%$

Cluster radius:

$\left[\begin{array}{lll}0.5 & 0.2 & 0.3\end{array}\right]$

Data range:

Error signal

Derivative error signal

Reference signal

Substractive clustering parameters: 
Quash factor

Accept ratio

Reject ratio
1.5

0.5

0.2

Using the substractive clustering algorithm, the resulted membership functions are shown in Figure 4. The PID controller with the substractive clustering based fuzzy HRC system is then simulated by using SIMULINK according to the block diagram in Figure 2. In this paper, the fuzzy HRC continuously sends the reference signal $(d(t))$ to manipulate the default reference signal $(r(t))$ as $\delta$ is set very small. The output response, the reference signal and the control signal are shown in Figure 5, it can be seen the PID - fuzzy HRC yields lower maximum overshoot of the output response and uses lower control signal than the standard PID controller as a result of applying manipulated reference signal (set-point). Other parameters selection leads to different transient performances.
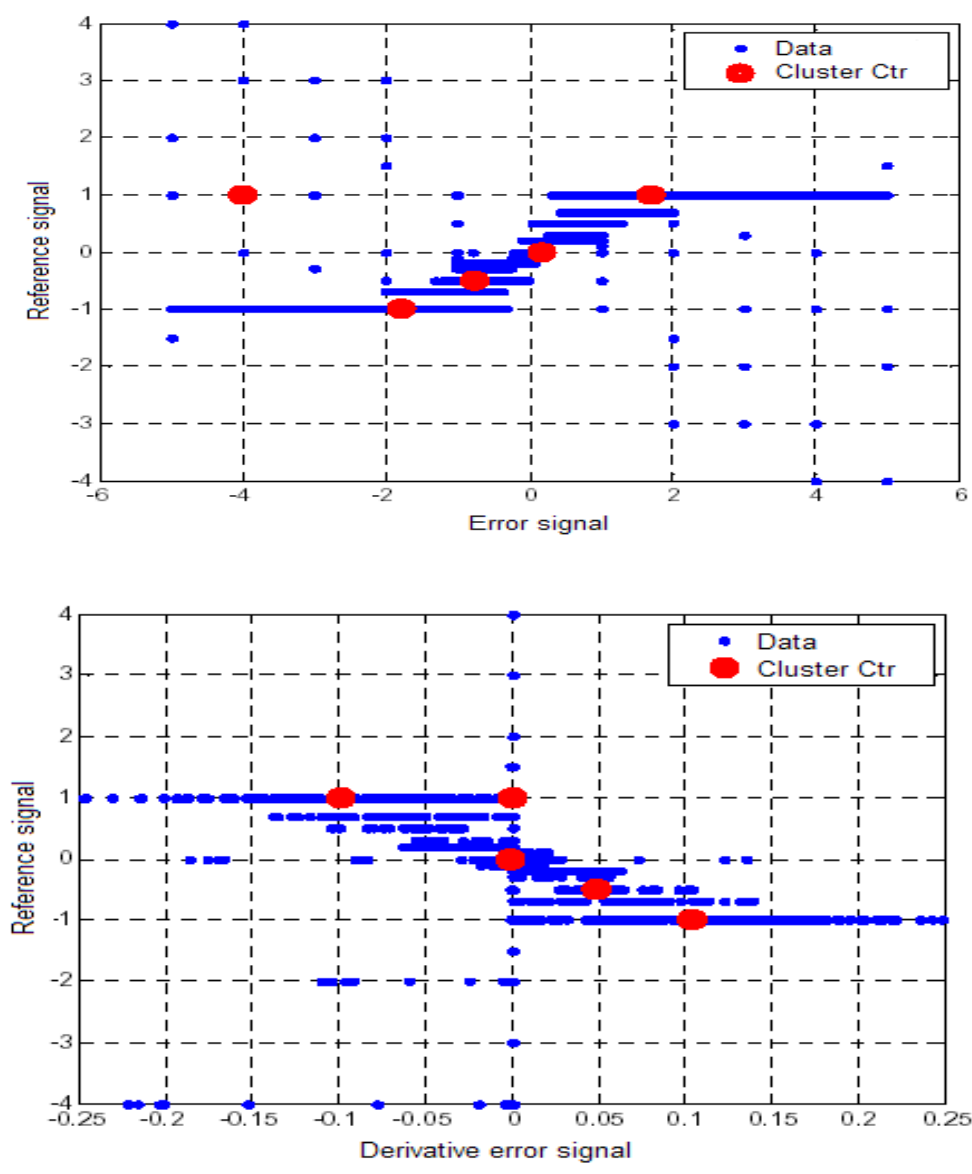


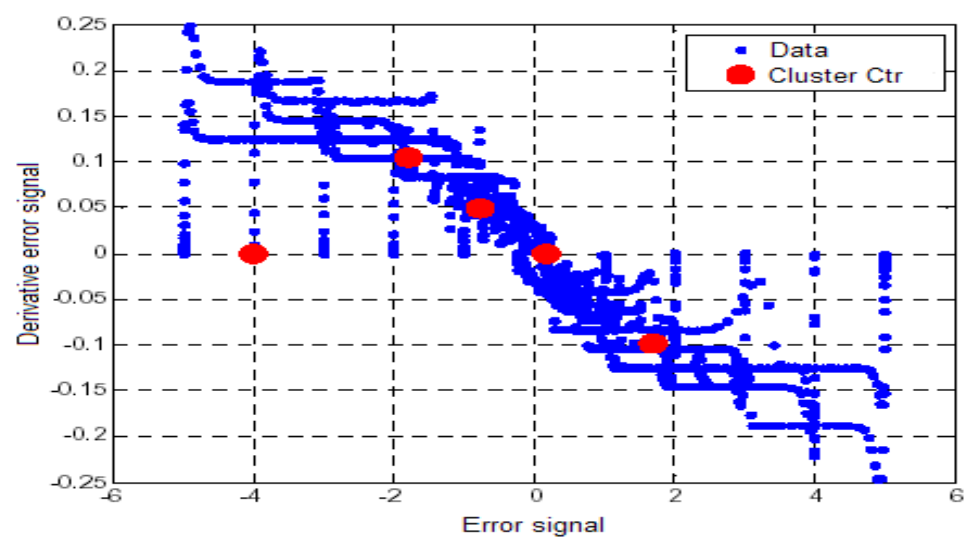

Figure 5 Data and cluster centers.
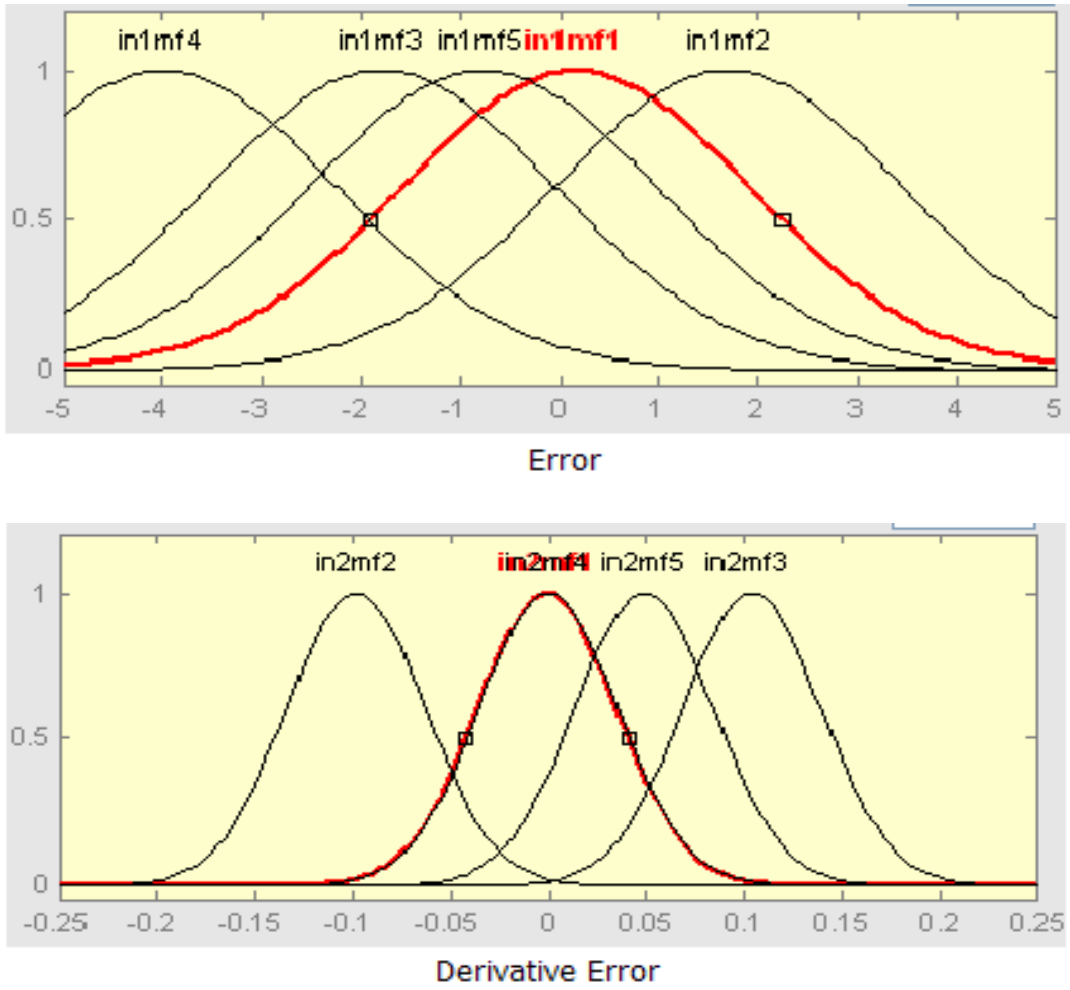

Figure 6 Membership functions. 



Figure 7 Output response and control signal of PID and PID-fuzzy HRC.

\section{Conclusions}

The paper proposed a method called fuzzy hybrid reference control (fuzzyHRC) which mimics the operator action in achieving the required transient response performance by increasing or decreasing the input of the controlled process by means of manipulating the reference signal. The combination of the proposed fuzzy HRC with the PID controller resulted in a two-degree-offreedom controller structure which is useful in solving conflicting performance requirements in one degree of freedom controller, such as transient performance and stability robustness. In this method, the stability property of the closed loop control system was determined by the parameter values of the PID controller. The fuzzy HRC system improved the transient response performance of the conventional closed loop system with PID controller without deteriorating the closed loop stability and it is achieved by using less control signal. The 
application of the substractive clustering technique provided a fast method to find the membership functions of the fuzzy HRC system. The proposed method can be extended to other stabilizing controllers, such that robust $\mathrm{H}_{\infty}$, robust dissipative.

\section{References}

[1] Joelianto, E. \& Williamson, D., Discrete Event Reference Control, Proc. $36^{\text {th }}$ IEEE Conference on Decision Control, 1, 692-697, 1997.

[2] Joelianto, E. \& Williamson, D., Optimal Full State Hybrid Reference Control, in Beghi, A., Finesso, L., and Picci, G. (Eds.), Mathematical Theory of Network Systems, Il Poligrafo, Padova, Italy, 941-944, 1998.

[3] Joelianto, E., Linear Hybrid Reference Control Systems, PhD Thesis, The Australian Nattional University, Canberra, Australia, 2000.

[4] Zadeh, L.A., Outline of A New Approach to The Analysis of Complex Systems and Decision Processes, IEEE Transactions on Systems, Man and Cybernetics, 3, 28-44, 1973.

[5] Wang, L.X., Adaptive Fuzzy Systems and Control: Design and Stability Analysis, Prentice Hall, Inc., Upper Saddle River, USA, 1994.

[6] Misir, D. \& Malki, H.A., Liapunov Stability for A Fuzzy PID Controlled Flexible-Joint Manipulator, International Journal of Computer Applications in Technology, 27, 97-106, 2006.

[7] Mohan, B.M. \& Sinha, A., Analytical Structure and Stability Analysis of A Fuzzy PID Controller, Applied Soft Computing Journal, 8, 749-758, 2008 .

[8] Yokogawa, UT14/UT14 Digital Indicating Controllers, Technical Information Publication TI 5B4A7-01E, Yokogawa Electric, Japan, 1990.

[9] Yokogawa, Green Series-Digital Indicating Controllers Selection Guides, Bulletin 5A1A01-E, Yokogawa Electric, Japan, 1998.

[10] Chiu, S., Developing Commercial Applications of Intelligent Control, IEEE Control Systems Magazine, 17, 94-97, 1997.

[11] Joelianto, E. \& Tansri, O., Fuzzy Logic Based Hybrid Reference Control for Improving Transient Response Performance of PID Controller, ITB Journal, 39A, 124-145, 2007. (In Indonesian)

[12] Takagi, T. \& Sugeno, M., Fuzzy Identification of Systems and Its Application to Modeling and Control, IEEE Transactions on Systems, Man and Cybernetics, 15, 116-132, 1985.

[13] Ziegler, J.G. \& Nichols, N.B., Optimum Setting for Automatic Controllers, Transactions ASME, 64, 759-768, 1942.

[14] Smith, C.A. \& Corripio, A.B., Principles and Practice of Automatic Process Control, John Wiley \& Sons, Inc., Canada., 1985.

[15] Coughanowr, D.R., Process Systems Analysis and Control, $2^{\text {nd }}$ Ed., McGraw-Hill Int. Edition, Singapore, 1991. 
[16] Åström, K.J. \& Hägglund, T., PID Controllers: Theory, Design, and Tuning, $2^{\text {nd }}$ Ed., Instrument Society of America, USA, 1995.

[17] Chiu, S.L., Fuzzy Model Identification Based on Cluster Estimation, Journal of Intelligent and Fuzzy Systems, 2, 267-278, 1994.

[18] Chiu, S.L., Extracting Fuzzy Rules from Data for Function Approximation and Pattern Classification, in Dubois, D., Prade, H., and Yager, R. (Eds.), Fuzzy Information Engineering: A Guided Tour of Applications. John Wiley \& Sons, Inc., New York, USA, 1997.

[19] Hammouda, K. \& Karray, F., A Comparative Study of Data Clustering Techniques, SYDE 625: Tools of Intelligent Systems Design, Course project, http://pami.uwaterloo.ca/ hammouda/publications.php (July 18, 2008), 2000.

[20] Fuzzy Logic Toolbox for Use with MATLAB, The MathWorks Inc., Natick, 2002.

[21] Joelianto, E. \& Williamson, D., Transient Response Improvement of Feedback Control Systems using Hybrid Reference Control, International Journal of Control, 82(10), 1955-1970, 2009.

[22] Francis, B.A. \& Wonham, W.M., The Internal Model Principle of Control Theory, Automatica, 12, 457-465, 1976,. 